\title{
Experimental Studies of the Turbulent Flow with Imposed Unsteadiness Structure
}

\author{
${ }^{1}$ Sabina M. Rzaeva, ${ }^{2}$ Lida A. Feoknistova, ${ }^{3}$ Tatiana V. Rzaeva, ${ }^{4}$ Irina P. Talipova \\ 1, 2,3,4 Kazan Federal University, Institute of Fundamental Mechanics of Liquid and Gas \\ *Email: aida_8691@ rambler.ru
}

Received: 21st October 2017 Accepted: 16th November 2017, Published: 31st December 2017

\begin{abstract}
The results of experimental studies of developed turbulent flow in a circular tube with superimposed flow pulsations are presented. They performed the measurements of time-averaged static pressure losses, instantaneous velocity values in the cross section of the pipe and pulsation pressure components over a wide frequency range of superimposed airflow pulsations. They determined a nonmonotonic dependence of static pressure variation change on the frequency of superimposed pulsations. New data are obtained on the spatial temporal characteristics of turbulent flow in a pipe with superimposed flow pulsations. The phase shifts between parameter pulsations are revealed.
\end{abstract}

Keywords: Turbulence, Pulsations, Hydrodynamic Non Stationarity, Flow Pulsations, Flow Structure

\section{Introduction}

Non stationary turbulent flows are often found in many technical devices the work of which is related to the flow of liquid and gas. Non-stationarity is the change of one or another flow characteristic in time. Turbulent flows are non-stationary flows a priori, since the parameters of the turbulent flow (velocity, temperature, etc.) vary in time randomly, even when the average values of these parameters are not changed. An example of nonstationary turbulent flows is the flow at the outlet of a piston pump, the flow on the suction and the exhaust of the internal combustion engine, the boundary layer on the surface of the gas turbine blade rotating in the tracks behind the stator blades, the flow in the combustion chamber at the start and operation of the jet engine, etc.

At present, the most the kinematic structure of movable turbulent boundary layers is the most studied one. There are experimental studies by Bukreev and Shakhin, Grigoriev and Fafurin, Ramapryan and Tu, Achary and Reynolds, Cousteau, Carlson, Parikh, Simpson, Hartner, Misushin, Shemer, Iguchi and others.

The systematic theoretical and experimental studies of the turbulent gas flow under the conditions of hydrodynamic nonstationarity were carried out by G.A. Dreicer and V.M. Kraev [1-3]. The study of currents with periodic flow pulsations is complicated by the essential dependence of the hydrodynamic parameter profiles on the frequency and the amplitude of the superimposed pulsations. However, the obtained data do not provide a complete picture of flow nonstationarity factor influence through the channel on the hydrodynamic parameters in general and the hydraulic resistance in particular.

The purpose of the present studies is to obtain the experimental data on the spatial-temporal kinematic structure of a turbulent flow in a circular tube with periodic flow nonstationarity.

\section{Experimental Equipment and Measuring Instruments}

The experiments were carried out in an experimental device, the scheme of which is shown on Fig. 1. The installation consisted of a working section 1 consisting of a round metal hydraulically smooth tube with an internal diameter of $64 \mathrm{~mm}$, the pulsator 2, the receiver 3 with a volume of more than $1 \mathrm{~m} 3$, a set of critical nozzles 4 and a turbocharger 5 . The pipe was divided into a pre-connected section, the length of which was 5 $\mathrm{m}$ (about 80 gauges in internal diameter), a measuring section of $2 \mathrm{~m}$ in length and a post connected section. The ends of the measuring section had fittings 6 for static pressure selection, fittings 7 for the connection of a pressure pulsation sensor, and coordinate device 8 with the probes of thermal-anemometric flow velocity sensor were installed. An average air flow by time in the installation was provided by the turbocharger 5 operating on the suction and kept constant within the same series of measurements with critical nozzles 4 installed on the airtight partition of the receiver 3 .

Pulsator 2 was a sealed compartment with a special device built into it to create superimposed pulsations of flow (speed) within the installation path in a wide range of amplitudes and pulsation frequencies. The flow pulsations were provided by periodical closing of the hole 9 (in the form of a triangle with rounded corners), an elliptical shutter 10 (Fig. 1, b) driven by an electric drive with an adjustable and stabilized speed. It was possible to change the degree of the hole 9 overlapping (distance d) in the pulsator design to vary the amplitude of the flow pulsation.

The static pressure sampling at the ends of the measuring section was taken through the hole with 0.8 $\mathrm{mm}$ diameter in a pipe wall. The pressure drop change across the measuring section was measured with a Ushaped manometer.

Each pneumatic circuit connecting the pressure extraction port to the manometer was provided with a 
damping section made of a metal tube with the length of $350 \mathrm{~mm}$ and an internal diameter of $0.8 \mathrm{~mm}$. This design of the pneumatic circuit provided the damping of pressure pulsations above the column of liquid and almost eliminated periodic mass exchange between the pneumatic tract and the installation path.
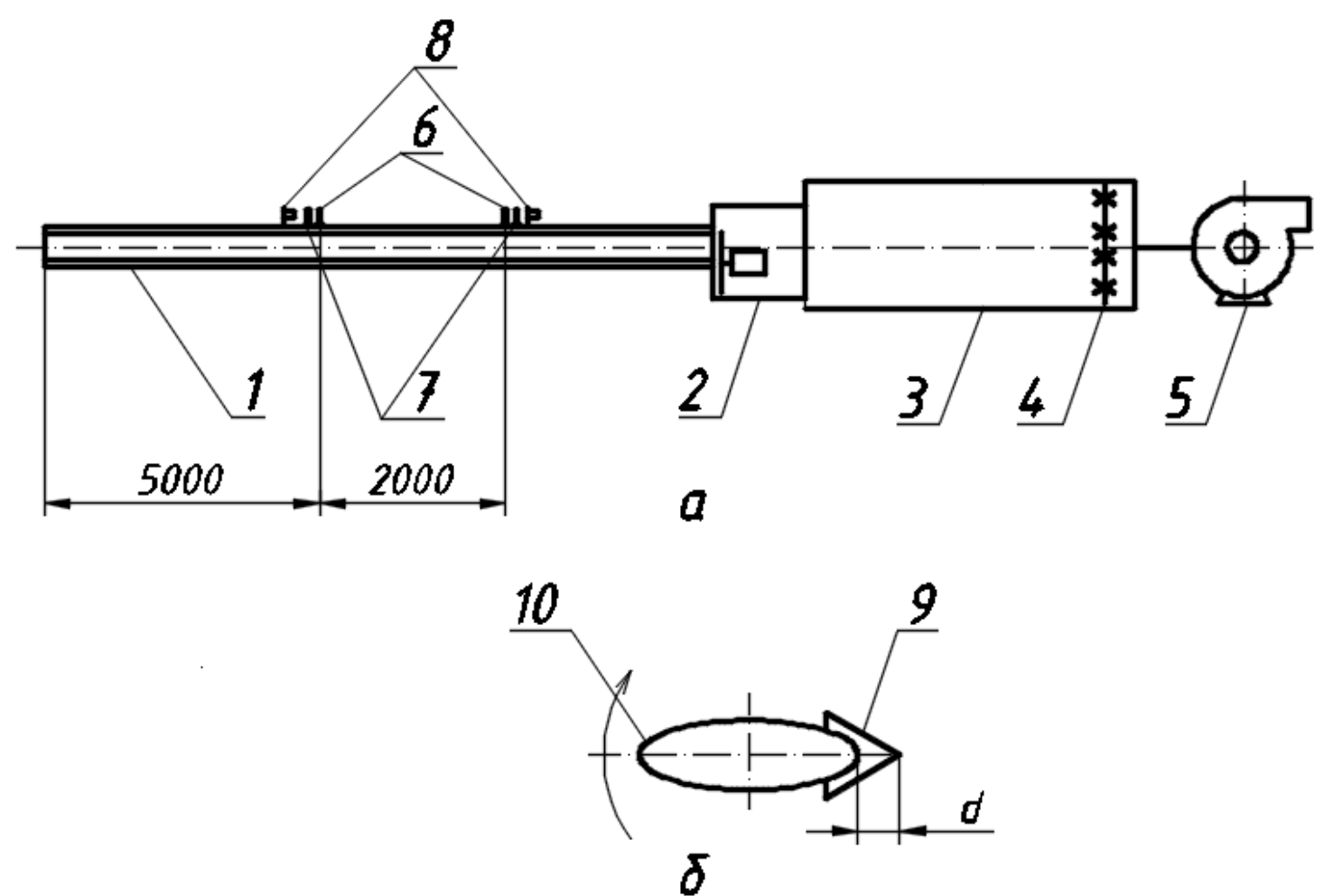

Fig. 1. Experimental Setup. The Main Operating Parameters of the Installation: - The Frequency of Superimposed Pulsations of Flow $\mathrm{f}=1-200 \mathrm{~Hz}$; - The Degree of Overlap of the Square of the Expenditure - Hole Pulsator d = $0-$ $100 \%$; - The Average Air Flow Path Q = $1-800 \mathrm{~m}^{3} / \mathrm{h}$.

The measurements of the static pressure drop at the measuring section were performed, and simultaneous measurements of the pulsating pressure components and flow velocity profiles were performed at the boundaries of the section. The pressure pulsations were measured using the microphones connected to the nozzles 7 (Fig. 1), and single-screw thermalanemometric sensors were used to measure the flow rate. These sensors moved along the radius of the pipe using a precision coordinate device.

The work of the microphones was supported by the RFT acoustic equipment, and the operation of speed sensors was carried out with a thermal anemometer unit of DISA 55M equipment. The collection and the processing of experimental data were performed using an automated system including a PC and an eightchannel analog-digital converter with parallel channel interrogation. When measurements were performed, the polling rate of the sensors was $5 \mathrm{kHz}$, the polling time was $2 \mathrm{~s}$.

\section{Results and Discussion}

Prior to the carrying out the research hydraulic resistance measurements were performed in pulsating currents with the turbulent flow in the pipe in a stationary mode for the values of the volumetric flow rate of air through critical nozzles $Q_{\mathrm{c}}=543.7 ; 380.6$ and $307,5 \mathrm{~m}^{3} / \mathrm{h}$ :

$$
\varsigma=(d / L) \Delta P_{c} /\left(\rho<U>^{2} / 2\right)
$$

where $d$ is the diameter of the pipe cross-section; $L$ is the length of the measuring section; $\Delta P_{\mathrm{c}}$ is the static pressure drop at the boundaries of the measuring section; $\langle U\rangle$ is the average flow rate; $\rho$ is the density.

The average flow rate was estimated from the flow rate through the nozzles, taking into account the pressure difference between the measuring section and the receiver. The similarity criterion is the Reynolds number calculated according to the average flow rate and the inner diameter of the pipe. In the experiments it was $\operatorname{ReQ}=\left(\begin{array}{llll}1.2 & \ldots & 2.0\end{array}\right) 10^{5}$. The results of the measurements showed that the difference in the value of $\zeta$ from the experimental Nikuradze data [4] for the developed turbulent pipe flow does not exceed $3-5 \%$. The measurements of the flow velocity profile were made in the measuring section of the device. The comparison of the measured profile with a known grade (according to the law 1/7) profile [5] showed their good agreement, which indicates the developed turbulent pipe flow in the measuring section. The mean square intensity profiles of the flow velocity pulsations have a maximum value near the wall, the value of which is $10 \%$ of the flow velocity on the tube axis, which is also agreed with the known experimental data. The experiments in pulsating flow were carried out with a maximum degree of the pulsator flow crosssection area change - in one turn of the flap 10 (Fig. 1, 
b), the area of the hole 9 opening section changed smoothly from 0 to $100 \%$ of the maximum area twice. The maximum area of the pulsator discharge opening is close to the area of the pipe cross-section. The analysis of oscillogram velocity showed that in the entire range under study the frequency variation of the flow velocity on the pipe axis changed according to the law close to the harmonic one. Considering the presence of some change in the amplitude of the velocity pulsation with respect to time and the deviation of oscillation form from the sinusoidal one, the intensity of the flow velocity pulsations was subsequently characterized not by the relative amplitude $A_{u} /<U>$, but by the magnitude of the relative mean square pulsations $\sigma_{u} /\langle U\rangle$, where $\sigma=\sqrt{\overline{\left(U^{\prime}\right)^{2}}}$ is the mean square value of speed pulsation. The evaluation $\sigma_{u} /\langle U\rangle$ is more stable in a statistical sense. For strictly harmonic oscillations, the relationship between the amplitude and the meansquare deviations is unambiguous.

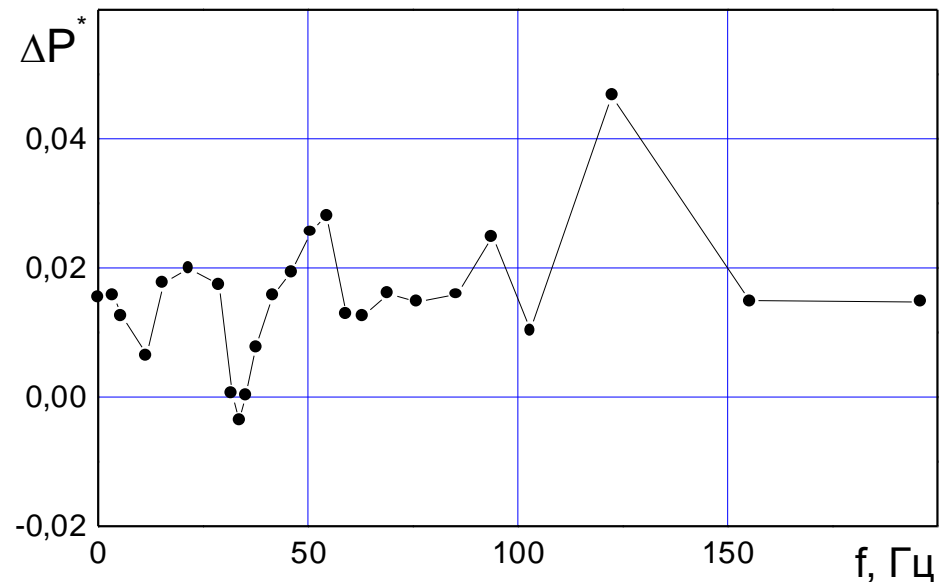

Fig. 2 Addiction Given Pressure Differential from the Frequency of Superimposed Pulsations of Flow when Qc= $386.13 \mathrm{~m} 3 / \mathrm{h}$

Figure 2 shows the dependence of the presented pressure drop on the measuring section of the pipe at the pulsating turbulent flow $\Delta P^{*}$ on the flow pulsation frequency for the average flow rate corresponding to the volumetric flow rate through the critical nozzles $Q_{\mathrm{c}}$ $=386.13 \mathrm{~m}^{3} / \mathrm{h}$. The presented pressure drop $\Delta P^{*}$ was determined according to the following formula:

$$
\Delta P^{*}=\frac{D}{l} \times \frac{\Delta P_{c}}{\rho \frac{<U>^{2}}{2}} .
$$

It can be seen from the figure that the nonmonotonic dependence of the pressure drop on the frequency is observed practically throughout the entire range of the flow pulsation frequency change. There are two expressed minima: at the frequency of $f=8-11 \underline{\mathrm{Hz}}$ and $f=33-35 \underline{\mathrm{Hz}}$. At $f=33-35 \underline{\mathrm{Hz}}$ the differential pressure takes a negative value, namely, the static pressure in the outlet section of the measuring pipe section becomes higher than in the inlet section. It should be noted that a similar change of $\Delta \mathrm{P}^{*}$ was observed at average flow rates $\mathrm{Q}_{\mathrm{c}}=307.5 \mathrm{~m}^{3} / \mathrm{h}$., $\mathrm{Q}_{\mathrm{c}}=$ $543.7 \mathrm{~m}^{3} / \mathrm{h}$ and it is well reproduced in repeated experiments.

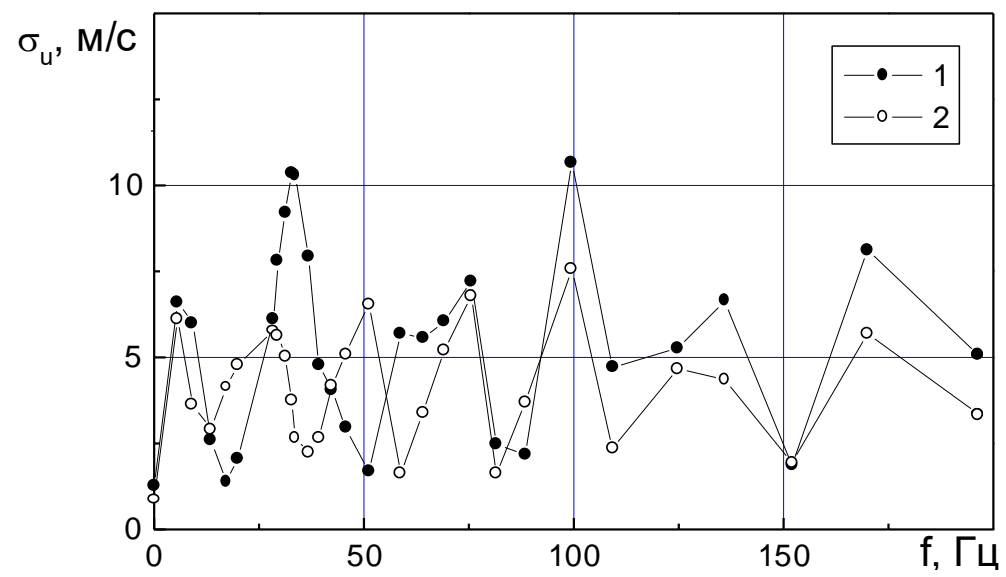

Copyright @ 2018 Helix ISSN 2319 - 5592 (Online) 
Fig. 3. The Dependency of the RMS Intensity of the Velocity Pulsations of the Flow at the Pipe Axis the Frequency at $\mathrm{QA}=386.13 \mathrm{~m}^{3} / \mathrm{s} ; 1$ - section 12 - section 2 .

The dependence of the mean-square intensity of the flow velocity pulsations within the tube axis on the frequency of superimposed pulsations in two sections of the measuring section, obtained from the results of simultaneous measurements, is shown on Fig. 3.

As can be seen from the figure, in some modes the minimum value of velocity pulsation intensity in the input section corresponds to the maximum intensity of pulsations in the output section of the working section, and vice versa by the frequency of flow superimposed pulsations. A similar nature is represented by the change of mean square pressure pulsations, however, the regions of the maximum value of $\sigma u$ (by the frequency of superimposed pulsations) coincide with the regions of minimum values of $\sigma p$, and vice versa. It should be noted that in some flow regimes the amplitude of the pressure pulsations can be sharply increased and can significantly exceed the value of the averaged velocity pressure in the pipe.

The obtained data testify to the existence of resonant phenomena at the pulsating flow in the experimental setup tract, which make a significant influence on the amplitude of flow parameter pulsations. At some resonant frequencies standing waves can exist, then there are antinodes and pressure (velocity) nodes, respectively in the corresponding sections of the tube at the maximum and minimum values of the amplitude of the pressure pulsations. Under the influence of flow pulsations, the deformation average velocity profile may take place in comparison with the velocity profile of a developed turbulent tubular flow at a steady state. The degree of this deformation in the case of resonant phenomena may be increased significantly. The consequence of these phenomena may be the appearance of a flow separation near the pipe wall. As the authors' studies showed [6-9], some regimes may have instantaneous return currents for a pulsed flow in a circular pipe - a short-time flow separation near the pipe wall. It was shown that the probability of reverse currents depends on the frequency and the amplitude of the flow velocity pulsations. The deformation of the average velocity profiles will lead to the change of movements in the cross sections considered. Consequently, the static pressure difference in these sections will not correspond to the hydraulic resistance of the channel.

For a more detailed description of the mechanism concerning the effect of superimposed non-stationarity on the spatial-temporal structure of the turbulent flow in a circular tube, the data analysis of pressure pulsation simultaneous measurements was performed in the inlet and the outlet sections of the measuring area, the pulsations and the flow velocity on the pipe axis in these sections, as well as flow velocities at different points of the pipe cross-section in each of these sections. Simultaneous measurements were made at the five most characteristic points according to the frequency of the flow superimposed pulsations: $\mathrm{f}=$ $24.8 ; 32.63 ; 52.4 ; 80$ and $123.2 \mathrm{~Hz}$.

It was established that the maximum value of pressure pulsation mutual correlation ratio in the input and output sections of the measuring area, as well as the coefficient of flow velocity pulsation mutual correlation on the channel axis in these same sections reach

0.95-0.98. 

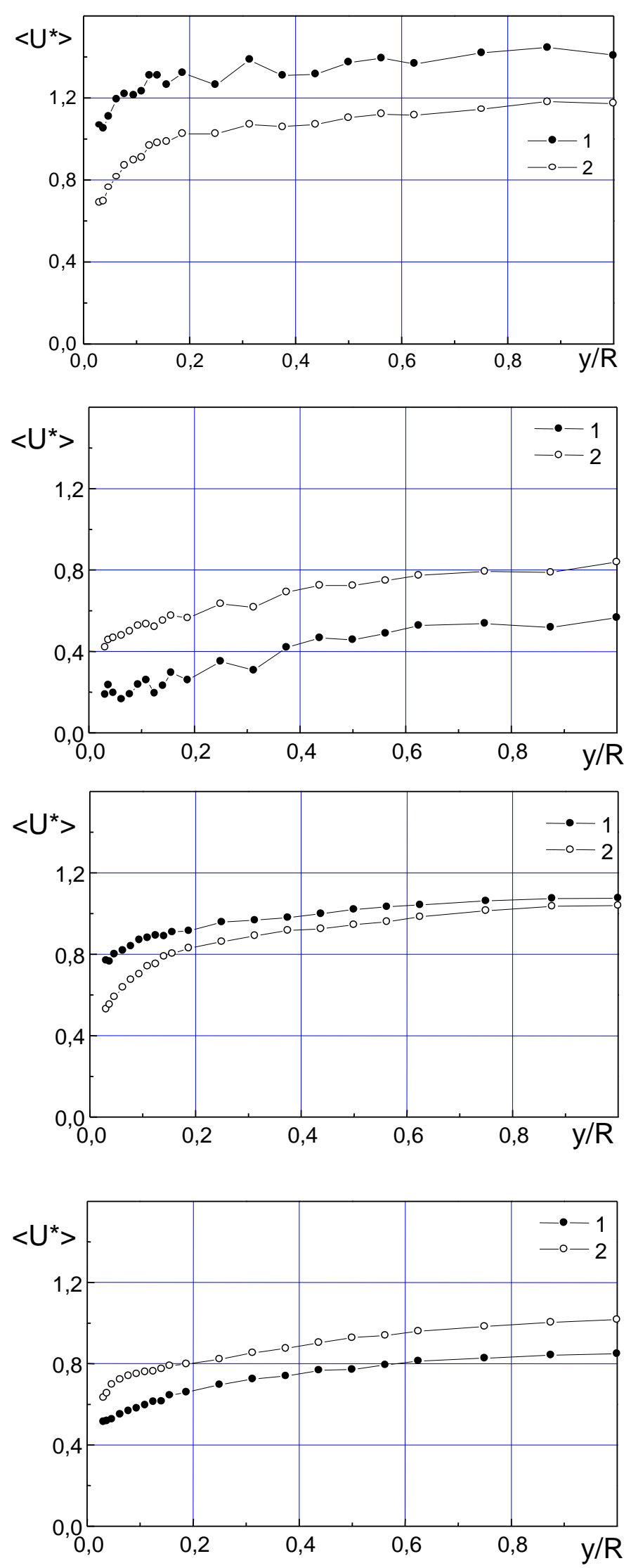

Fig.4. Conditionally Averaged Velocity Profiles: $\mathrm{a}-\varphi=90^{\circ}, \mathrm{b}-\mathrm{f}=32.6 \mathrm{~Hz}, \varphi=270^{\circ} \mathrm{c}-\varphi=90^{\circ}$, $\mathrm{g}-\mathrm{f}=52.4 \mathrm{~Hz}, \varphi=270^{\circ}: 1-$ section $1 ; 2-$ section 2 .
The phase shift is detected between the pulsations of these parameters, the magnitude of which varies nonmonotonically with respect to the frequency of superimposed pulsations and in some modes (by frequency) it reaches the following values: 0,2 for velocity pulsations, and 0,5 for pressure pulsations of superimposed pulsation period. Consequently, the law of air motion in the working area of the device under the influence of flow pulsations differs significantly from the "law of a solid body motion".

A close relationship is also found between the pressure pulsations and the flow velocity at all points of each pipe section. The value of the cross-correlation coefficient between the pulsations of these parameters $R_{u-p}$ reaches $0.9-0.95$ on the axis of the tube, depending on a mode (according to the frequency of superimposed pulsations) and decreases slightly near the wall to $\mathrm{R}_{\mathrm{u}-\mathrm{p}}=0.8-0.9$.

$b \quad$ Besides, there is a phase shift between the pressure pulsations and the flow velocity, the magnitude and sign of which varies depending on the frequency of the superimposed pulsations and the position of the section in which the measurements

$c \quad$ were made (the input and output sections of the measuring area). This indicates a complex spatial-temporal structure of the flow, a more detailed study of which requires additional research. However, there is some change in the phase shift between the pressure pulsations and the flow velocity in all cases when you approach the pipe wall (approximately

$g \quad 0.05-0.1$ of the period). In other words, the pulsations of the flow velocity near the wall outstrip the pulsations on the tube axis, i.e. the change of speed at different pipe points does not occur simultaneously. The data obtained contradict the results of [10], in which it is asserted that under the influence of superimposed pulsations of velocity its change at all points of the pipe section occurs by phase. Perhaps the reason for such a contradiction lies in the small value of the phase shift, which the authors of [10] were not allowed to determine due to the means of measurement used in the experiments. 

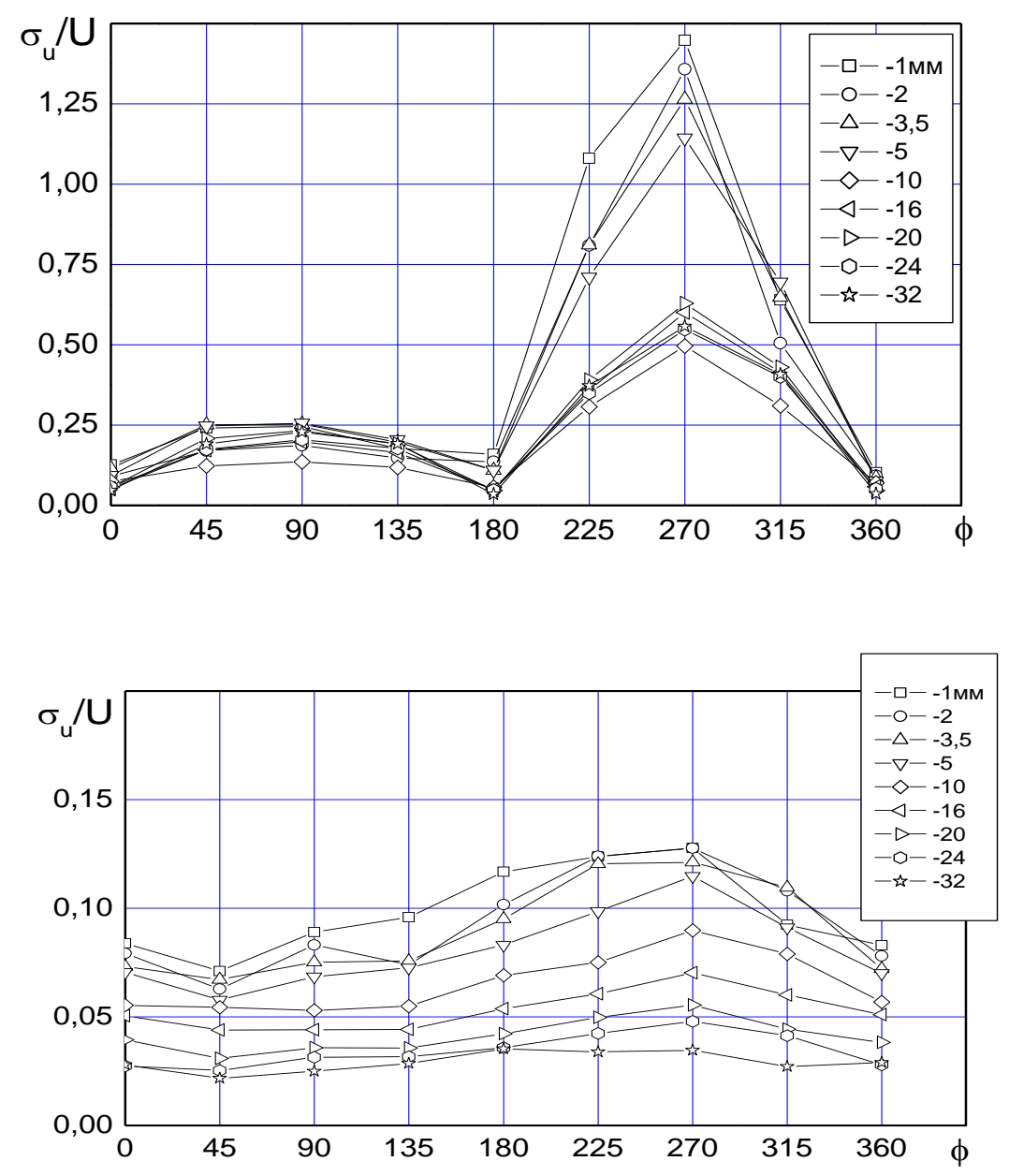

$b$

Fig. 5. The Change in the Intensity of Turbulent Fluctuations of Velocity in Cross Section 1 at the Phase Angle at Different Distances from the Wall of a $-\mathrm{f}=32.6 \mathrm{~Hz}, \mathrm{~b}-\mathrm{f}=52.4 \mathrm{~Hz}$.

According to the experiment results, conditionally averaged profiles of the flow velocity in the inlet and outlet sections of the measured pipe section were obtained, as well as the change velocity pulsation intensity at various distances from the pipe wall. The conditional averaging was carried out according to the oscillogram of the pressure variation in the corresponding sections. The binding of measurement parameter results on the wall and at various distances from the wall to the phase of the pressure change allows one to present the experimental data in the form of space-time fields of conditional mean values and the intensity of hydrodynamic parameter turbulent pulsations. Some of the conditionally averaged velocity profiles are shown on Fig. 4, and the distribution of velocity pulsation intensity for a number of flow regimes is shown on Fig. 5. The analysis of the data obtained shows that the velocity profiles becomes less filled in the phase of pressure buildup than in the phase of pressure decrease. A similar regularity was noted in [11]. The intensity of velocity pulsations in the phase of pressure increase expands, its most intensive growth is observed near the wall of the tube. At a frequency of superimposed pulsations $\mathrm{f}=33.6 \mathrm{~Hz}$, where the negative values of the pressure drop at the measuring section take place, the velocity profile in the vicinity of the phase angle $\phi \approx 270^{\circ}$ has a nonmonotonic character near the wall, and the mean flow velocity is significantly reduced. The analysis of flow velocity oscillograms in the section of 1 measuring section in this flow regime showed that at some points in time the velocity value becomes close to 0 . In the same region of phase angle $\phi$ variation the intensity of the velocity pulsations increases sharply and makes $140 \%$, which is explained by the influence of two factors: the increase of mean square velocity pulsation $\overline{\left(U^{\prime}\right)^{2}}$ as the wall is approached and a simultaneous decrease of the averaged $U$ motion velocity.

The abovementioned data support the assumption of a short-term flow separation occurrence near the pipe wall in some regimes according to the frequency of superimposed pulsations.

\section{Conclusions}

The experimental data about spatial-temporal structure of a turbulent flow in the tube with periodic nonstationarity are obtained. A nonmonotonic dependence of pressure drop change on the frequency 
of superimposed pulsations is established. The phase shifts between the pulsations of the parameters are revealed. It is established that in some flow regimes the amplitude of pressure pulsations exceeds the averaged pressure velocity. It is shown that the velocity profile becomes less filled in the phase of pressure increase, and the intensity of the velocity pulsations increases, and in certain regimes the probability to cause shortterm flow separation near the pipe wall is high according to the frequency of superimposed pulsations.

\section{Acknowledgements}

The work is performed according to the Russian Government Program of Competitive Growth of Kazan Federal University.

\section{References}

1. Dreitser G.A., Kraev V.M. Turbulent flow structure, heat transfer and hydrodynamic at hydrodynamic unsteady conditions reserach // Izv. RAN. Energetika. 2006. № 4. P. 131144.

2. G.A. Dreitser, V.M. Kraev. The study of frequency pulsation spectra during the flow in a pipe under nonstationary conditions. // Abstracts from reports of the Vth Minsk International forum on heat and mass transfer, May 24-28, 2004 Minsk, ITMO. - 2004. - pp. 69-70.

3. V.M. Kraev and A.I. Tikhonov. "On the physical principles of hydrodynamic nonstationarity effect on turbulent flow" // The Bulletin of Samara State Aerospace University, No. 4 (20), 2009, pp. 128-135.

4. Nikuradse J. Gesetzmassgkeiten der turbulenten Stromung in glatten Rohren. VDI, Forschungsheft, 1932, Bd. 356.

5. Schlichting G. The theory of the boundary layer. M.: Science. 1974. 711 p.

6. Kozlov A.P. Mikheyev N.I. Stinski G.V. Zanko F.S. Interaction of hydrodynamic and heat processes in turbulent separated flows // ASME Int. Mech. Eng. Conf. and Exhibition (IMECE 2002) Nov. 17-22, 2002.- New Orlean, Lousiana, - IMEGE2002-33609. pp.115-122

7. Goltsman A.E., Davletshin I.A., Mikheev N.I., Paereliy A.A., «Shear Stresses in Turbulent Pulsating Channel Flow», Thermophys. Aeromechanics, 22:3 (2015), pp. 319-328

8. Mikheev A.N., Mikheev N.I., Molochnikov V.M. "Experimental evaluation of flow characteristics in a pulsating flow visualization device", AkademEnergo proceedings, 2013, no. 1, 27-37

9. Goltsman A.E., Davletshin I.A., Kolchin S.A., Mikheev N.I., «Flow Rate Through a Long Channel with a Rotating Damper At the End», Fluid Dyn., 49:4 (2014), 443-446

10. Koval'nogov N.N. Flow structure and the features of turbulent exchange in a boundary layer of a dynamically unsteady flow within channels // RAS bulletin.

11. Markov S.B. Experimental research of flow structure and hydraulic resistance in unsteady turbulent flows // Mekhanika szidkosti I gasa. 1973. № 2. pp. 65-75. 